\title{
Stable cGVHD
}

National Cancer Institute

\section{Source}

National Cancer Institute. Stable CGVHD. NCI Thesaurus. Code C103142.

No change in chronic Graft versus Host Disease (cGVHD) progression during the preceding 3 months. 